УДК 517.956.4

S. D. IVASYShen, I. P. MEDYNSKY

\title{
ON APPLICATIONS OF THE LEVI METHOD IN THE THEORY OF PARABOLIC EQUATIONS
}

\begin{abstract}
S. D. Ivasyshen, I. P. Medynsky. On applications of the Levi method in the theory of parabolic equations, Mat. Stud. 47 (2017), 33-46.

A short review of papers where a fundamental solution was constructed by the Levi's parametrix method is done. Some details of applications of this method for parabolic equations and systems of parabolic equations, in particular the procedure of constructing a parametrix for equations with different degenerations and singularities, are considered. The basic results of constructing of a fundamental solution for degenerate ultraparabolic equations of the Kolmogorov type by the Levi paramerix method under different assumptions on the coefficients are reviewed. Some new authors' results on the constructing of a classical fundamental solution and, so-called, $L$-fundamental solution for the equation with coefficients with two groups of spatial variables are presented. These results are obtained by stepwise using of the Levi parametrix method and special Hölder conditions on the coefficients.
\end{abstract}

1. Notations and definitions. Let $n$ and $N$ be given natural numbers; $|k|:=\sum_{j=1}^{n} k_{j}$ if $k:=\left(k_{1}, \ldots, k_{n}\right) \in \mathbb{Z}_{+}^{n}$. Below we shall consider a one-dimensional variable $t$ and an $n$ dimensional variable $x:=\left(x_{1}, \ldots, x_{n}\right) \in \mathbb{R}^{n} ; t$ and $x_{1}, \ldots, x_{n}$ will be interpreted as the time and spatial variables, respectively. Denote by $\Pi_{H}:=\left\{(t, x) \mid t \in H \subset \mathbb{R}, x \in \mathbb{R}^{n}\right\}$ a layer and by $T$ a fixed positive number, by $\mathbb{C}_{N 1}$ and $\mathbb{C}_{N N}$ the sets of all $N \times 1$ or $N \times N$ matrices with complex entries.

Let us consider the equation

$$
L_{N}\left(t, x, \partial_{t}, \partial_{x}\right) u(t, x)=0, \quad(t, x) \in \Pi_{(0, T]},
$$

Here $L_{N}\left(t, x, \partial_{t}, \partial_{x}\right)$ is a linear differential expression with complex-valued coefficients depending on $t$ and $x,(t, x) \in \Pi_{[0, T]}$. It is a scalar if $N=1$ or $N \times N$-matrix if $N>1 ; u: \Pi_{(0, T]} \rightarrow$ $\mathbb{C}_{N 1}$ is an unknown function.

Suppose that a differential expression $L_{N}$ is uniformly parabolic in the sense of Petrovsky or of Eidelman on the layer $\Pi_{[0, T]}$.

The fundamental solution of the Cauchy problem (FSCP) is defined as follows.

Definition 1. A FSCP for the equation (1) is such a function

$$
Z(\cdot, \cdot ; \tau, \xi): \Pi_{(\tau, T]} \rightarrow \mathbb{C}_{N N}
$$

2010 Mathematics Subject Classification: 35A09, 35K10, 35K65, 35K70.

Keywords: parabolic equations with degeneration; fundamental solutions; the Levi parametrix method; ultraparabolic equations of the Kolmogorov type.

doi:10.15330/ms.47.1.33-46

(C) S. D. Ivasyshen, I. P. Medynsky, 2017 
depending on the parametric point $(\tau, \xi) \in \Pi_{[0, T)}$, that the formula

$$
u(t, x)=\int_{\mathbb{R}^{n}} Z(t, x ; \tau, \xi) \varphi(\xi) d \xi, \quad(t, x) \in \Pi_{(\tau, T]},
$$

determines a solution of the equation $(1)$ on $\Pi_{(\tau, T]}$, satisfying the initial condition

$$
\left.u\right|_{t=\tau}=\varphi
$$

for any $\tau \in[0, T)$ and an arbitrary continuous bounded function $\varphi$.

In other words, FSCP is a function (2) such that for any $(\tau, \xi) \in \Pi_{[0, T)}$ it is the solution of the Cauchy problem

$$
L_{N} Z=0,\left.\quad Z\right|_{t=\tau}=I_{N} \delta_{\xi}
$$

where $I_{N}$ is the $N \times N$ unit matrix and $\delta_{\xi}$ is the Dirac delta-function concentrated in the point $\xi$.

2. An essence of the Levi method. In 1907 Italian mathematician E. E. Levi published the paper [1]. In this paper the fundamental solution for elliptic equation of $2 n$-th order with coefficients depending on two independent variables was constructed. The method proposed by E. E. Levi reduces the problem of constructing of the fundamental solution to the problem of solving a spacial integral equation. In 1941 the paper of Levi was translated in Russian ([2]), and in 1946 his result was generalized by Z. Ya. Shapiro [3] for three variables.

During the period 1946-1963 Ya. B. Lopatynsky lived and worked in Lviv. One of the main research directions of this period was construction and investigation of properties of fundamental matrices for general elliptic systems of the partial differential equations. In the paper [4] Ya. B. Lopatynsky proved the existence of the fundamental matrix of solutions for a general elliptic system by using the Levi method. After this fundamental paper of Ya. B. Lopatynsky other mathematicians are engaged in construction of fundamental matrices of solutions. Research papers of Ya. B. Lopatynsky stimulated also research in the theory of the parabolic systems. To read more about the influence of ideas of Ya. B. Lopatynsky on the development of the theory of the parabolic systems it is possible in the paper of S. D. Ivasyshen ([5]).

A FSCP for second order parabolic equations with bounded variable coefficients (depending on all the variables) was constructed by F. Dressel [6] for the smooth coefficients, and by W. Pogorzelski [7] and D. G. Aronson [8] for the Hölder continuous coefficients. FSCPs for systems of equations of arbitrary orders were constructed by S. Z. Bruk, S. D. Eidelman, W. Pogorzelski, L. N. Slobodetsky, D. G. Aronson and M. I. Matiychuk.

The FSCPs constructed and investigated in the above papers found a variety of applications in the studies of properties of parabolic systems, correct solvability of the Cauchy problem in various classes of functions, integral representations of solutions of the Cauchy problem, as well as solutions defined on the open layer $\Pi_{(0, T]}$, local solvability of the Cauchy problem for quasilinear and nonlinear parabolic (in the sense of Petrovsky) systems, extension of solutions of such systems onto larger time intervals, etc. These results are expounded in detail in the monographs $[9,10]$. Similar results for the parabolic in the sense of Eidelman systems of equations and also for the parabolic systems with degeneration on the initial hyperplane and with bounded or increasing coefficients were obtained. The Levi parametrix method was used for the construction of FSCP. 
An essence of the method Levi is as follows. In this method a FSCP $Z(t, x ; \tau, \xi)$ is sought as a sum of the principal term $Z_{0}(t, x ; \tau, \xi)$ that is called a parametrix, and an additional summand $W(t, x ; \tau, \xi)$ having the integral form with the kernel $Z_{0}$ and some unknown density $Q$. The parametrix $Z_{0}$ has a desired singularity at $(t, x)=(\tau, \xi)$. Usually it is defined on the basis of the FSCP $G_{0}(t, x ; \tau, \xi ; y)$ or $G_{0}(t, x ; \tau, \xi ; \beta, y)$ for model equations containing only principal (in a certain sense) groups of terms. In their coefficients the variables $x$ or $t, x$ are fixed in the points $y$ or $(\beta, y)$ respectively. FSCP $G_{0}$ is determined with the use of the Fourier transform. The Fourier transform in spatial variables is applied to a model equation. In order to obtain the parametrix, in the expression for $G_{0}$ one should substitute the appropriate functions, that may depend on the main variables $t, x$ and the parametric variables $\tau, \xi$ instead of $y$ or $\beta, y$. Unknown density $Q$ is determined from an integral equation of the Volterra type (unlike to an elliptic case) on the variable $t$ with quasiregular kernel and could be solved by the method of successive approximations.

3. Determination of a parametrix. We shall present some examples of choosing of a parametrix for parabolic systems.

Suppose that $L_{N}\left(t, x, \partial_{t}, \partial_{x}\right)=I_{N} \partial_{t}-\sum_{|k| \leq 2 b} a_{k}(t, x) \partial_{x}^{k}$. In this case the model equations with coefficients that depend on $t$ and parameter $y \in \mathbb{R}^{n}$ or only parameters $(\beta, y) \in \Pi_{[0, T]}$, have the form

$$
\left(I_{N} \partial_{t}-\sum_{|k|=2 b} a_{k}(t, y) \partial_{x}^{k}\right) u=0
$$

or

$$
\left(I_{N} \partial_{t}-\sum_{|k|=2 b} a_{k}(\beta, y) \partial_{x}^{k}\right) u=0
$$

respectively.

Let $G_{0}(\cdot, \cdot ; \cdot, \cdot ; y)$ and $G_{0}(\cdot, \cdot ; \cdot, \cdot ; \beta, y)$ are FSCPs for equations (5) and (6), respectively. Then the function

$$
Z_{0}(t, x ; \tau, \xi):=G_{0}(t, x ; \tau, \xi ; \xi), 0 \leq \tau<t \leq T,\{x, \xi\} \subset \mathbb{R}^{n} .
$$

is the parametrix for the equation (5). We construct the parametrix for the equation (6) in a similar way. We have

$$
Z_{0}(t, x ; \tau, \xi):=G_{0}(t, x ; \tau, \xi ; \tau, \xi), 0 \leq \tau<t \leq T,\{x, \xi\} \subset \mathbb{R}^{n} .
$$

Let us consider the parabolic system with degeneration on the initial hyperplane. In this case we have $L_{N}\left(t, x, \partial_{t}, \partial_{x}\right)=\alpha(t) I_{N} \partial_{t}-\beta(t) \sum_{0<|k| \leq 2 b} a_{k}(t, x)-a_{0}(t, x)$. Here $\alpha(t)>0$, $\beta(t)>0$ are positive functions in $t \in(0, T], \alpha(0) \beta(0)=0$ and $\beta$ is a monotonously nondecreasing function. The parametrix is defined by the formula $(7)$, where $G_{0}(\cdot, \cdot ; \cdot, \cdot ; y)$ is a FSCP for the system

$$
\left(\alpha(t) I_{N} \partial_{t}-\beta(t) \sum_{|k|=2 b} a_{k}(t, y) \partial_{x}^{k}-a_{0}(t, y)\right) u=0 .
$$

In this case a model equation contains not only major terms but also minor (according to the order of differentiation) terms, that is caused with the structure of the system. An analogical situation appears in the case of equation with growing coefficients. For example, let us consider such equation

$$
\left(\partial_{t}-S_{b}-\sum_{|k| \leq 2 b} a_{k}(t, y) \partial_{x}^{k}\right) u=0
$$


where $S_{b}:=b \sum_{j=1}^{n} \partial_{x_{j}}\left(x_{j} u\right), b \in \mathbb{R}$. The model equation for finding of function $G_{0}$ in this case has the form

$$
\left(\partial_{t}-S_{b}-\sum_{|k|=2 b} a_{k}(t, y) \partial_{x}^{k}\right) u=0
$$

The additional summands (such as $S_{b}$ ) make the process of constructing of FSCP and its structure more complicated. And if we investigate the properties of a parametrix and of potentials generated by this parametrix, then we have the additional difficulties. The successful choice of the parametrix allows to obtain the exact estimates of FSCP and of its derivatives and find their differences.

4. Degenerate equations of the Kolmogorov type. Below we consider different generalizations of the known Kolmogorov's equation of diffusion with inertia [11]. If $n=1$, then this equation has the form

$$
\partial_{t^{\prime}} g=-\dot{q}^{\prime} \partial_{q^{\prime}} g-\partial_{\dot{q}^{\prime}}\left(f\left(t^{\prime}, q^{\prime}, \dot{q}^{\prime}\right) g\right)+\partial_{\dot{q}^{\prime}}^{2}\left(k\left(t^{\prime}, q^{\prime}, \dot{q}^{\prime}\right) g\right),
$$

where $q, \dot{q}$ are the coordinates of an one-dimensional physical system at an instant of time $t$ and $q^{\prime}, \dot{q}^{\prime}$ are the coordinates of this system at an arbitrary instant $t^{\prime}>t$. A.N. Kolmogorov constructed a FSCP for this equation if the coefficients $f$ and $k$ are constants.

The equation of diffusion with inertia is degenerated equation because the leading terms of a such equation contain only the derivatives that correspond to basic spatial variables. Such equations with the second order differential operator are called the ultraparabolic equations. Subsequent investigations aimed at finding as weak conditions as possible for the existence of FSCP, obtaining its precise estimates, considering equations with a more complicated structure. Different classes of equations that generalize the Kolmogorov equation are considered in the monograph [10]. Note that their generalizations may contain not only one group of degeneration, but they may contain several groups of degeneration.

In this paper we shall consider only the equations with one group of degeneration. Let $n_{1}, n_{2}$ be given natural numbers, $n_{1} \geq n_{2} \geq 1, n=n_{1}+n_{2}$. So that in this case the spatial variable $x \in \mathbb{R}^{n}$ has the form $x:=\left(x_{1}, x_{2}\right), x_{j}:=\left(x_{j 1}, \ldots, x_{j n_{j}}\right) \in \mathbb{R}^{n_{j}}, j \in\{1,2\}$. Denote by $k:=\left(k_{1}, k_{2}\right) \in \mathbb{Z}_{+}^{n}$ corresponding multi-indices, where $k_{j}:=\left(k_{j 1}, \ldots, x_{j n_{j}}\right) \in \mathbb{Z}_{+}^{n_{j}}, j \in\{1,2\}$. We shall say that $t$ and $x_{1}$ are basic variables and $x_{2}$ is the variable of group of degeneration. In this case ultraparabolic equation of the Kolmogorov type has the form

$$
L_{1}\left(t, x, \partial_{t}, \partial_{x}\right) u:=\left(S-A\left(t, x, \partial_{x_{1}}\right)\right) u=0,
$$

where

$$
S:=\partial_{t}-\sum_{j=1}^{n_{2}} x_{1 j} \partial_{x_{2 j}}, \quad A\left(t, x, \partial_{x_{1}}\right):=\sum_{j, l=1}^{n_{1}} a_{j l}(t, x) \partial_{x_{1 j}} \partial_{x_{1 l}}+\sum_{j=1}^{n_{1}} a_{j}(t, x) \partial_{x_{1 j}}+a_{0}(t, x)
$$

is a linear second order differential operator with respect to $x_{1}$ with complex valued coefficients $a_{j l}, a_{j}$ and $a_{0}$, that depend on $(t, x) \in \Pi_{[0, T]}$.

Let us describe shortly the papers where FSCP was constructed for ultraparabolic equations of the Kolmogorov type under different assumptions on the coefficients.

M. Weber ([12]) constructed a FSCP for the equation

$$
\sum_{i, j=1}^{n} a_{i j}(t, x, y) \partial_{x_{i}} \partial_{x_{j}} u+\sum_{i=1}^{n} a_{i}(t, x, y) \partial_{x_{i}} u+a(t, x, y) u+\sum_{i=1}^{n} x_{i} \partial_{y_{i}} u+\partial_{t} u=0,
$$


on an arbitrary open subset of the phase space.

In the paper [13] A. M. Il'in stated the existence, uniqueness, and positivity of a FSCP for the equation

$\left(\partial_{t}-\sum_{i, j=1}^{n} a_{i j}(t, x, y) \partial_{x_{i}} \partial_{x_{j}}-\sum_{i=1}^{n} a_{i}(t, x, y) \partial_{x_{i}}-a(t, x, y)-\sum_{i=1}^{m} b_{i}(t, x, y) \partial_{y_{i}}\right) u=f, \quad m \leq n$,

where the coefficients $a_{i j}, a_{i}, a$ and $b_{i}$ are continuous and bounded together with their derivatives of the order up to two and four respectively, as well as a theorem on the uniqueness of a solution of the Cauchy problem in the Tikhonov class, and inner estimates of solutions.

I. M. Sonin ([14]) used the Levi method to construct a FSCP for the equation

$\left(\partial_{t}-\frac{1}{2} \sum_{i, j=1}^{n} a_{i j}(t, x, y, z) \partial_{y_{i}} \partial_{y_{j}}-\sum_{i=1}^{n} a_{i}(t, x, y, z) \partial_{y_{i}}-\sum_{j=1}^{n} b_{j}(t, x, y, z) \partial_{x_{j}}-\sum_{j=1}^{n} c_{j}(t, x, y, z) \partial_{z_{j}}\right) u=0$,

where the coefficients $a_{j l}$ and $a_{j}$ are bounded on $[0, T] \times \mathbb{R}^{3 n}$ together with their derivatives on all the variables of the order up to two and one respectively, while $b_{j}$ and $c_{j}$ (the coefficients $c_{j}$ do not depend on $y$ ) have bounded derivatives of the orders up to three, where $b_{j}(t, x, y, z)$ and $c_{j}(t, x, y, z)$ behave "approximately" like $y_{j}$ and $x_{j}$, respectively. A FSCP for such equation is the transition density for some degenerate diffusion process (so-called process of Sonin). More information about applications FSCPs in the theory of diffusion processes, in the physics and in the financial mathematics could be found in the survey papers [15, 16].

L. P. Kuptsov studied the equations of the form

$$
\left(\partial_{t}-\sum_{j, l=1}^{n} a_{j l}(t) \partial_{x_{j}} \partial_{x_{l}}+\sum_{j, l=1}^{n} b_{j l}(t) x_{j} \partial_{x_{l}}\right) u=0
$$

where $\left(a_{j l}(t)\right)_{j, l=1}^{n}$ is non-negative symmetric matrix of rank $n_{1}\left(1 \leq n_{1} \leq n\right)$, the matrix $\left(b_{j l}(t)\right)_{j, l=1}^{n}$ has a special structure and properties. A FSCP for the general equation (10) was constructed in paper [17].

The papers of S. D. Eidelman, S. D. Ivasyshen, H. P. Malytska, L. M. Tychynska, L. M. Androsova, V. S. Dron' and V. V. Layuk ([18-27]) are devoted to the construction of FSCPs and their applications for the ultraparabolic equations of the second or any orders.

The series of papers [28-31] of Italian mathematicians is devoted to equations of the Kolmogorov type that have the form

$$
L u:=\sum_{i, j=1}^{p_{0}} a_{i j}(t, x) \partial_{x_{i}} \partial_{x_{j}} u+\sum_{i=1}^{p_{0}} a_{i}(t, x) \partial_{x_{i}} u+c(t, x) u+\sum_{i, j=1}^{p} b_{i j} x_{i} \partial_{x_{j}} u-\partial_{t} u=0,
$$

where $1 \leq p_{0}<p, A_{0}:=\left(a_{i, j}\right)_{i, j=1}^{p_{0}}$ is a symmetric matrix, positively defined for each $(t, x) \in \Pi_{[0, T]}$, the matrix $B:=\left(b_{i, j}\right)_{i, j=1}^{p}$ has constant real elements and it has the form

$$
\left(\begin{array}{ccccc}
* & B_{1} & O & \ldots & O \\
* & * & B_{2} & \ldots & O \\
\ldots & \ldots & \ldots & \ldots & \ldots \\
* & * & * & \ldots & B_{r} \\
* & * & * & \ldots & *
\end{array}\right) .
$$


Here $B_{j}$ is a $p_{j-1} \times p_{j}$ matrix of rank $p_{j}$, where $p_{0}, p_{1}, \ldots, p_{r}$ are positive integer numbers such as $p_{0} \geq p_{1} \geq \ldots \geq p_{r} \geq 1, p_{0}+p_{1}+\ldots+p_{r}=p$, and $*$ denotes an arbitrary blocks. The class of such equations is wider then the class of ultraparabolic equations of the Kolmogorov type. Under certain assumptions on the matrix (12), the operator $L$, defined by the formula (11), is hypoelliptic and invariant with respect to a certain dilation group. S. Polidoro introduced a special definition for the $B$-Hölder continuity of functions that corresponds to above group. In the case when the coefficients $a_{i j}, a_{i}$ and $c$ are $B$-Hölder continuous with the use of the results of the papers $[17,29]$ a FSCP for the equation (11) is constructed by the Levi method in [29-33]. Its estimates, the property of normality, the formula of convolution, and also some theorems on existing a solution of the Cauchy problem, uniqueness and the integral representation for non-negative solutions are established. A solution of the equation (11) is the function $u$, which has continuous ordinary derivatives $\partial_{x_{i}} u, \partial_{x_{i}} \partial_{x_{j}} u,\{i, j\} \subset$ $\left\{1, \ldots, p_{0}\right\}$, and Lie-derivative $Y u$ with respect to the vector field which defined by the following differential expression

$$
\sum_{i, j=1}^{p} b_{i j} x_{i} \partial_{x_{j}}-\partial_{t}
$$

and in each point $(t, x)$ such function satisfies the equation

$$
\sum_{i, j=1}^{p_{0}} a_{i j}(t, x) \partial_{x_{i}} \partial_{x_{j}} u+\sum_{i=1}^{p_{0}} a_{i}(t, x) \partial_{x_{i}} u+c(t, x) u+Y u=0 .
$$

So, constructed in the papers of Italian mathematicians FSCPs are non classical FSCPs. Such solution is called a $L$-FSCP.

In the monograph [10] there were obtained the conditions on coefficients of the degenerate equation of Kolmogorov type (not only ultraparabolic but also equations of arbitrary order or equations that are parabolic with respect to basic variables in the sense of Eidelman) that allow to construct a $L$-FSCP, to find the estimates of the FSCP and of its derivatives with respect to basic spatial variables and derivative $S u$.

Similarly to the case of non-degenerate parabolic equations it is possible to obtain a complete analytic description of a FSCP, which leads to very precise results on the correct solvability of the Cauchy problem and integral representations for solutions, for the Kolmogorov type equations with constant coefficients or coefficients depending only on time variable. If coefficients of a Kolmogorov type equation depend on all variables, then it is much more complicated to study its FSCP. In addition to usual difficulties of the Levi method, new serious difficulties are caused by the degeneracy of the equations. Because the operator $S$ "weave together" the spatial and the time variables, the process of obtaining exact estimates of a FSCP become complicated. This difficulty is overcame in the monograph [10] with the help of a special condition for the coefficients. In the case of the equation (9) this condition has the form

$$
\begin{array}{r}
\exists H>0 \quad \exists \alpha \in(0,1] \quad \forall\{(t, x),(\tau, \xi)\} \subset \Pi_{[0, T]}: \\
|a(t, x)-a(\tau, \xi)| \leq H(d(t, X(t-\tau) ; \tau, \xi))^{\alpha} .
\end{array}
$$

Here $X(t):=\left(X_{1}(t), X_{2}(t)\right), \quad X_{1}(t):=x_{1}, X_{2}(t):=x_{2}+t \hat{x}_{1}, \hat{x}_{1}:=\left(x_{11}, \ldots, x_{1 n_{2}}\right)$, $d(t, x ; \tau, \xi):=|t-\tau|^{1 / 2}+\left|x_{1}-\xi_{1}\right|+\left|x_{2}-\xi_{2}\right|^{1 / 3}$ and $a$ is $a_{j l}$ or $a_{j}$ or $a_{0}$. 
In this case the parametrix is defined by the formula (8), where $G_{0}(\cdot, \cdot ; \cdot, \cdot ; \beta, y)$ is the FSCP for the below equation with parameters

$$
\left(S-A\left(\beta, y, \partial_{x_{1}}\right)\right) u=0 .
$$

The another approach is used in the papers of H.P. Malytska ([18, 20, 21]). The coefficients of the equation satisfy the ordinary conditions (they are continuous on $t$, differentiable and have Hölder continuous derivatives with respect to spatial variables). The model equation for finding FSCP has the form

$$
\left.\left(S-A(t, y), \partial_{x_{1}}\right)\right) u=0 .
$$

If $G_{0}(\cdot, \cdot ; \cdot, \cdot ; y)$ is a FSCP for the equation (15), then a parametrix $Z_{0}$ is defined by the formula $Z_{0}(t, x ; \tau, \xi)=G_{0}(t, x ; \tau, \xi ; \Xi(t-\tau))$, where $\Xi(t):=\left(\xi_{1}, \xi_{2}-t \hat{\xi}_{1}\right)$. And further, on the stage of applying of the Levi method H. P. Malytska does not takes in to account the complicated dependence of the parametrix on the parameters at the calculation of $L_{1} Z_{0}$, that does the explanation of the method wrong.

5. Classical FSCP for equations of the Kolmogorov type. The analysis of the papers showed that a decision of the problem of constructing of the classical FSCP consists not only in choice of suitable conditions on the coefficients but also in the successful choice of a parametrix for using of the Levi method. Our approach is based on step-by-step use of the Levi method. On the first stage a FSCP for the equation with coefficients depending on the basic variables and the parameter (or the parameters) is constructed. An amount of the parameters is equal to the amount of the groups of degeneration in the equation. At the second stage of constructing FSCP we choose the above FSCP as the parametrix. An amount of stages of the constructing of FSCP depends on the quontity of the groups of spatial variables in the equation. Such approach is realized in the paper [32]. Its basic results are presented below.

Hereafter we use the notations

$$
\begin{gathered}
\Delta_{x}^{z} f(\cdot, x, \cdot):=f(\cdot, x, \cdot)-f(\cdot, z, \cdot) ; \quad \Delta_{x_{1}}^{z_{1}} f(\cdot, x, \cdot)=\Delta_{x}^{z^{(1)}} f(\cdot, x, \cdot):= \\
:=f(\cdot, x, \cdot)-f\left(\cdot, z^{(1)}, \cdot\right) ; \Delta_{x_{2}}^{z_{2}} f(\cdot, x, \cdot)=\Delta_{x}^{z^{(2)}} f(\cdot, x, \cdot):=f(\cdot, x, \cdot)-f\left(\cdot, z^{(2)}, \cdot\right) ; \\
z^{(1)}:=\left(z_{1}, x_{2}\right), z^{(2)}:=\left(x_{1}, z_{2}\right) ;\left\{x, z, z^{(s)}\right\} \subset \mathbb{R}^{n},\left\{x_{s}, z_{s}\right\} \subset \mathbb{R}^{n_{s}}, s \in\{1,2\} .
\end{gathered}
$$

Let us consider the equation (9) under the following assumptions on the coefficients:

1) the coefficients $a_{j l}, a_{j}$ and $a_{0}$ are complex valued functions on $\Pi_{[0, T]}$, they are bounded and continuous on $t$ and there exists such constant $\delta>0$, that for any points $(t, x) \in \Pi_{[0, T]}$ and $\sigma_{1}:=\left(\sigma_{11}, \ldots, \sigma_{1 n_{1}}\right) \in \mathbb{R}^{n_{1}}$ the inequality

$$
\operatorname{Re} \sum_{j, l=1}^{n_{1}} a_{j l}(t, x) \sigma_{1 j} \sigma_{1 l} \geq \delta\left|\sigma_{1}\right|^{2} ;
$$

is true.

2) they fulfils Hölder conditions with respect to spatial variables in the following sense:

$$
\begin{gathered}
\exists H_{1}>0 \forall\left\{(t, x),\left(t, z^{(1)}\right)\right\} \subset \Pi_{[0, T]}:\left|\Delta_{x_{1}}^{z_{1}} a(t, x)\right| \leq H_{1}\left|x_{1}-z_{1}\right|^{\alpha_{1}}, \\
\exists H_{2}>0 \forall\left\{(t, x),\left(t, z^{(2)}\right)\right\} \subset \Pi_{[0, T]} \forall h \in[0, T]:
\end{gathered}
$$




$$
\left|\Delta_{x_{2}}^{z_{2}} a(t, x)\right| \leq H_{2}\left(h^{3 \alpha_{2} / 2}+\left|X_{2}(h)-z_{2}\right|^{\alpha_{2}}\right),
$$

were $a$ is equal to $a_{j l}$ or $a_{j}$ or $a_{0}, \alpha_{1} \in(0,1)$ and $\alpha_{2} \in(0,2 / 3)$.

If $h=0$ then the ordinary Hölder condition with respect to variable $x_{2}$ is implied from (18). The sufficient condition of implementation (18) gives the following lemma from the paper [32].

Lemma 1. Let $a$ is a continuous and bounded function on $\Pi_{[0, T]}$ be satisfing the condition

$$
\begin{gathered}
\exists H_{3}>0 \quad \exists \alpha \in(1 / 2,1] \quad \forall\left\{(t, x),\left(t, z^{(2)}\right)\right\} \subset \Pi_{[0, T]}: \\
\left|\Delta_{x_{2}}^{z_{2}} a(t, x)\right| \leq H_{3}\left(T^{1 / 2}+\left|\hat{x}_{1}\right|\right)^{-\alpha}\left|x_{2}-z_{2}\right|^{\alpha} .
\end{gathered}
$$

Then the inequality (18) is true with $\alpha_{2}=2 \alpha / 3$.

Thus, on the first stage we constract a FSCP for the equation

$$
\left(S-A\left(t,\left(x_{1}, y_{2}\right), \partial_{x_{1}}\right)\right) u(t, x)=0,(t, x) \in \Pi_{(0, T]}, y_{2} \in \mathbb{R}^{n_{2}},
$$

in the form

$$
\begin{gathered}
Z_{1}\left(t, x ; \tau, \xi ; y_{2}\right)=Z_{0}\left(t, x ; \tau, \xi ; y_{2}\right)+W_{1}\left(t, x ; \tau, \xi ; y_{2}\right), \\
W_{1}\left(t, x ; \tau, \xi ; y_{2}\right):=\int_{\tau}^{t} d \beta \int_{\mathbb{R}^{n}} Z_{0}\left(t, x ; \beta, \lambda ; y_{2}\right) Q_{1}\left(\beta, \lambda ; \tau, \xi ; y_{2}\right) d \lambda,
\end{gathered}
$$

where $Z_{0}$ is parametrix, and $Q_{1}$ is an unknown function.

For a parametrix we choose the function

$$
Z_{0}\left(t, x ; \tau, \xi ; y_{2}\right)=G_{0}\left(t, x ; \tau, \xi ;\left(\xi_{1}, y_{2}\right)\right), 0 \leq \tau<t \leq T,\{x, \xi\} \subset \mathbb{R}^{n}, y_{2} \in \mathbb{R}^{n_{2}}
$$

where $G_{0}(\cdot, \cdot ; \cdot, \cdot ; y)$ is a FSCP for the equation $\left(S-A\left(t, y, \partial_{x_{1}}\right)\right) u(t, x)=0$.

Since we consider the equation with two groups of spatial variables, the second stage is the last. We shall find the FSCP for the equation (9) in the form

$$
Z(t, x ; \tau, \xi)=Z_{2}(t, x ; \tau, \xi)+W_{2}(t, x ; \tau, \xi)
$$

where the function

$$
Z_{2}(t, x ; \tau, \xi)=Z_{1}\left(t, x ; \tau, \xi ; \xi_{2}\right), 0 \leq \tau<t \leq T,\{x, \xi\} \subset \mathbb{R}^{n}
$$

is a paramtrix, which is constructed by a FSCP $Z_{1}$, and

$$
W_{2}(t, x ; \tau, \xi):=\int_{\tau}^{t} d \beta \int_{\mathbb{R}^{n}} Z_{2}(t, x ; \beta, \lambda) Q_{2}(\beta, \lambda ; \tau, \xi) d \lambda,
$$

where $Q_{2}$ is an unknown function. The main result of the paper [32] is in the following theorem. 
Theorem 1. Let the conditions 1 and 2 with $\alpha_{1} \in(0,1)$ and $\alpha_{2} \in(1 / 3,2 / 3)$ be satisfied. Then there exists a classical FSCP $Z$ for the equation (9), and

$$
\begin{gathered}
\left|\partial_{x}^{k} Z(t, x ; \tau, \xi)\right| \leq C(t-\tau)^{-M-M_{k}} E_{c}(t-\tau, x, \xi), \\
|S Z(t, x ; \tau, \xi)| \leq C(t-\tau)^{-M-1} E_{c}(t-\tau, x, \xi),
\end{gathered}
$$

where $0 \leq \tau<t \leq T,\{x, \xi\} \subset \mathbb{R}^{n}, k=\left(k_{1}, k_{2}\right) \in \mathbb{Z}_{+}^{n},\left|k_{1}\right|+2\left|k_{2}\right| \leq 2, M:=n_{1} / 2+$ $3 n_{2} / 2, M_{k}:=\left|k_{1}\right| / 2+3\left|k_{2}\right| / 2, E_{c}(t, x, \xi):=\exp \left\{-c\left(t^{-1}\left|X_{1}(t)-\xi_{1}\right|^{2}+t^{-3}\left|X_{2}(t)-\xi_{2}\right|^{2}\right)\right\}, C$ and $c$ are some positive constants.

The fact that the Hölder exponent from the condition $\mathbf{2}$ with respect to the second spatial variable is smaller then $2 / 3$, corresponds to the results of Ya. I. Shatyro [34, 35] for model degenerate equations.

6. Existence of an $L$-FSCP. Let the condition 1 and the condition $\mathbf{2}$ with $\alpha_{1} \in(0,1)$ and $\alpha_{2} \in(0,1 / 3]$ be satisfied. Now we shall prove that there exists an $L$-FSCP $Z$ for the equation (9). For this purpose it is enough to get the existence of the Lie-derivative of the function $Z$. We shall remind the definition of the Lie-derivative ([28, 30]).

Definition 2. A function $u$ is differentiable in the sense of Lie at the point $(t, x)$ with respect to a vector field, that is defined by the differential expression $S$, if there exists the finite limit

$$
\left(S_{L} u\right)(t, x)=\lim _{h \rightarrow 0} h^{-1}(u(\gamma(t, x, h))-u(\gamma(t, x, 0)),
$$

where $\gamma(t, x, h):=(t+h, X(h)), h \in \mathbb{R}$ is the integral curve, which passes through the point $(t, x)$.

A limit $\left(S_{L} u\right)(t, x)$ is called the Lie derivative of the function $u$ at the point $(t, x)$ with respect to a given vector field.

Note that, if there exist the derivatives $\partial_{t} u, \partial_{x_{2}} u$ at the point $(t, x)$, then $\left(S_{L} u\right)(t, x)=$ $(S u)(t, x)$.

Since the function $Z$ is defined in the formula (23) and for a parametrix $Z_{2}$ (as it implies from Lemma 2 from [32]) there exists the $S Z_{2}$ and it has the estimate

$$
\left|S Z_{2}(t, x ; \tau, \xi)\right| \leq C(t-\tau)^{-M-1} E_{c}(t-\tau, x, \xi),
$$

it is enough to prove the existence of the Lie derivative of a volume potential $W_{2}$, which is defined in the formula (26). For this purpose consider such set of functions that depends on the parameter $\varepsilon$ :

$$
W_{2}^{\varepsilon}(t, x ; \tau, \xi):=\int_{\tau}^{t-\varepsilon} d \beta \int_{\mathbb{R}^{n}} Z_{2}(t, x ; \beta, \lambda) Q_{2}(\beta, \lambda ; \tau, \xi) d \lambda, 0<\varepsilon<\tau<t \leq T,\{x, \xi\} \subset \mathbb{R}^{n} .
$$

We write

$$
\begin{gathered}
h^{-1}\left(W_{2}^{\varepsilon}(t+h, X(h) ; \tau, \xi)-W_{2}^{\varepsilon}(t, x ; \tau, \xi)\right)= \\
=\int_{\tau}^{t-\varepsilon} d \beta \int_{\mathbb{R}^{n}} h^{-1}\left(Z_{2}(t+h, X(h) ; \beta, \lambda)-Z_{2}(t, x ; \beta, \lambda)\right) Q_{2}(\beta, \lambda ; \tau, \xi) d \lambda+ \\
+\int_{t-\varepsilon}^{t+h-\varepsilon} d \beta \int_{\mathbb{R}^{n}} h^{-1} Z_{2}(t+h, X(h) ; \beta, \lambda) Q_{2}(\beta, \lambda ; \tau, \xi) d \lambda=: J_{1}^{h}+J_{2}^{h}
\end{gathered}
$$


and find the limits of the integrals $J_{1}^{h}, J_{2}^{h}$ as $h \rightarrow 0$. We have

$$
\lim _{h \rightarrow 0} J_{1}^{h}=\int_{\tau}^{t-\varepsilon} d \beta \int_{\mathbb{R}^{n}} S Z_{2}(t, x ; \beta, \lambda) Q_{2}(\beta, \lambda ; \tau, \xi) d \lambda .
$$

If in the integral $J_{2}^{h}$ we shall make the change of a variable by the formula $\gamma=h^{-1}(\beta-t+\varepsilon)$, then

$$
J_{2}^{h}=\int_{0}^{1} d \gamma \int_{\mathbb{R}^{n}} Z_{2}(t+h, X(h) ; h \gamma+t-\varepsilon, \lambda) Q_{2}(h \gamma+t-\varepsilon, \lambda ; \tau, \xi) d \lambda,
$$

and

$$
\lim _{h \rightarrow 0} J_{2}^{h}=\int_{\mathbb{R}^{n}} Z_{2}(t, x ; t-\varepsilon, \lambda) Q_{2}(t-\varepsilon, \lambda ; \tau, \xi) d \lambda .
$$

So, we have

$$
\begin{aligned}
& S_{L} W_{2}^{\varepsilon}(t, x ; \tau, \xi)=\int_{\mathbb{R}^{n}} Z_{2}(t, x ; t-\varepsilon, \lambda) Q_{2}(t-\varepsilon, \lambda ; \tau, \xi) d \lambda+ \\
& +\int_{\tau}^{t-\varepsilon} d \beta S_{L} \int_{\mathbb{R}^{n}} Z_{2}(t, x ; \beta, \lambda) Q_{2}(\beta, \lambda ; \tau, \xi) d \lambda=: K_{21}^{\varepsilon}+K_{22}^{\varepsilon} .
\end{aligned}
$$

Taking into account the property of $Z_{2}$, we get

$$
\lim _{\varepsilon \rightarrow 0} K_{21}^{\varepsilon}=Q_{2}(t, x ; \tau, \xi), 0 \leq \tau<t \leq T,\{x, \xi\} \subset \mathbb{R}^{n}
$$

In order to prove the possibility of using of the operator $S_{L}$ under the signs of integrals in $K_{22}^{\varepsilon}$, let us consider the integral

$$
L(t, \beta, x):=\int_{\mathbb{R}^{n}} S Z_{2}(t, x ; \beta, \lambda) Q_{2}(\beta, \lambda ; \tau, \xi) d \lambda .
$$

We use the representation

$$
\begin{gathered}
L(t, \beta, x)=\int_{\mathbb{R}^{n}}\left(\sum_{j, l=1}^{n_{1}} \Delta_{x_{2}}^{\lambda_{2}} a_{j l}(t, x) \partial_{x_{1 j}} \partial_{x_{1 l}} Z_{2}(t, x ; \beta, \lambda)+\sum_{j=1}^{n_{1}} \Delta_{x_{2}}^{\lambda_{2}} a_{j}(t, x) \partial_{x_{1 j}} Z_{2}(t, x ; \beta, \lambda)+\right. \\
\left.\quad+\Delta_{x_{2}}^{\lambda_{2}} a_{0}(t, x) Z_{2}(t, x ; \beta, \lambda)\right) Q_{2}(\beta, \lambda ; \tau, \xi) d \lambda-\sum_{j, l=1}^{n_{1}} a_{j l}(t, x) \times \\
\times \int_{\mathbb{R}^{n}} \partial_{x_{1 j}} \partial_{x_{1 l}} Z_{2}(t, x ; \beta, \lambda) Q_{2}(\beta, \lambda ; \tau, \xi) d \lambda-\sum_{j=1}^{n_{1}} a_{j}(t, x) \int_{\mathbb{R}^{n}} \partial_{x_{1 j}} Z_{2}(t, x ; \beta, \lambda) Q_{2}(\beta, \lambda ; \tau, \xi) d \lambda- \\
\left.-a_{0}(t, x) \int_{\mathbb{R}^{n}} Z_{2}(t, x ; \beta, \lambda)\right) Q_{2}(\beta, \lambda ; \tau, \xi) d \lambda=: \sum_{k=1}^{4} L_{k}(t, \beta, x)
\end{gathered}
$$


valid due to the the definition of $Z_{2}$. In order to estimate the summand $L_{1}(t, \beta, x)$, we use the inequalities (19), (21) from [32] and the condition (18) with $h=t-\tau$. We have

$$
\begin{gathered}
\left|L_{1}(t, \beta, x)\right| \leq C \int_{\mathbb{R}^{n}}\left((t-\beta)^{-M-1}+(t-\beta)^{-M-1 / 2}+(t-\beta)^{-M}\right) \times \\
\times\left(|t-\beta|^{3 \alpha_{2} / 2}+\left|X_{2}(t-\beta)-\lambda_{2}\right|^{\alpha_{2}}\right) E_{c}(t-\beta, x, \lambda)(\beta-\tau)^{-M-1+3 \alpha_{2} / 2} E_{c}(\beta-\tau, \lambda, \xi) d \lambda \leq \\
\leq C((t-\beta)(\beta-\tau))^{-1+3 \alpha_{2} / 2}((t-\beta)(\beta-\tau))^{-M} \int_{\mathbb{R}^{n}} E_{c_{1}}(t-\beta, x, \lambda) E_{c}(\beta-\tau, \lambda, \xi) d \lambda \leq \\
\leq C(t-\tau)^{-M}((t-\beta)(\beta-\tau))^{-1+3 \alpha_{2} / 2} E_{c_{2}}(t-\tau, x, \xi), \\
0 \leq \tau \leq \beta<t \leq T,\{x, \xi\} \subset \mathbb{R}^{n}, c_{2}<c_{1}<c .
\end{gathered}
$$

By using the inequality (19) from [32], taking into account the boundedness of the coefficients of the equation, we obtain

$$
\begin{gathered}
\left|L_{3}(t, \beta, x)\right| \leq C(t-\beta)^{-M-1 / 2}(\beta-\tau)^{-1+3 \alpha_{2} / 2}((t-\beta)(\beta-\tau))^{-M} \times \\
\times \int_{\mathbb{R}^{n}} E_{c_{1}}(t-\beta, x, \lambda) E_{c}(\beta-\tau, \lambda, \xi) d \lambda \leq C(t-\tau)^{-M}(t-\beta)^{-1 / 2}(\beta-\tau)^{-1+3 \alpha_{2} / 2} \times \\
\times E_{c_{1}}(t-\tau, x, \xi), \quad 0 \leq \tau \leq \beta<t \leq T, \quad\{x, \xi\} \subset \mathbb{R}^{n}, \quad c_{1}<c, \\
\left|L_{4}(t, \beta, x)\right| \leq C(t-\beta)^{-M}(\beta-\tau)^{-1+m_{2} \alpha_{2}}((t-\beta)(\beta-\tau))^{-M} \times \\
\times \int_{\mathbb{R}^{n}} E_{c_{1}}(t-\beta, x, \lambda) E_{c}(\beta-\tau, \lambda, \xi) d \lambda \leq C(t-\tau)^{-M}(\beta-\tau)^{-1+3 \alpha_{2} / 2} \times \\
\times E_{c_{1}}(t-\tau, x, \xi), \quad 0 \leq \tau \leq \beta<t \leq T, \quad\{x, \xi\} \subset \mathbb{R}^{n}, \quad c_{1}<c .
\end{gathered}
$$

For the summand $L_{2}(t, \beta, x)$ we shall write such representation:

$$
\begin{gathered}
L_{2}(t, \beta, x)=-\sum_{j, l=1}^{n_{1}} a_{j l}(t, x) \int_{\mathbb{R}^{n}} \partial_{x_{1 j}} \partial_{x_{1 l}} Z_{2}(t, x ; \beta, \lambda) \Delta_{\lambda}^{X(t-\beta)} Q_{2}(\beta, \lambda ; \tau, \xi) d \lambda- \\
-\sum_{j, l=1}^{n_{1}} a_{j l}(t, x)\left(\int_{\mathbb{R}^{n}} \partial_{x_{1 j}} \partial_{x_{1 l}} Z_{2}(t, x ; \beta, \lambda) d \lambda\right) Q_{2}(\beta, X(t-\beta) ; \tau, \xi)=: \\
=: L_{21}(t, \beta, x)+L_{22}(t, \beta, x) .
\end{gathered}
$$

Estimations of the summands from (37) are carried out analogously to the estimation of the volume potentials (see the proof of Lemma 4) from the paper [33], with replacing $Z_{0}$ by $Z_{2}$ and $Q$ by $Q_{2}$, respectively. We obtain

$$
\begin{gathered}
\left|L_{21}(t, \beta, x)\right| \leq C(t-\tau)^{-M}(t-\beta)^{-1+\alpha_{1}^{0} / 2}(\beta-\tau)^{-1+3 \alpha_{2} / 2-\alpha_{1}^{0} / 2} E_{c_{1}}(t-\tau, x, \xi), \\
\left|L_{22}(t, \beta, x)\right| \leq C(t-\tau)^{-M}(t-\beta)^{-1+\alpha_{1} / 2}(\beta-\tau)^{-1+3 \alpha_{2} / 2} E_{c_{1}}(t-\tau, x, \xi) .
\end{gathered}
$$


Formulas (37), (38) and (39) imply the estimate:

$$
\left|L_{2}(t, \beta, x)\right| \leq C(t-\tau)^{-M}(t-\beta)^{-1+\alpha_{1}^{0} / 2}(\beta-\tau)^{-1+3 \alpha_{2} / 2-\alpha_{1}^{0} / 2} E_{c_{1}}(t-\tau, x, \xi) .
$$

Here $0 \leq \tau \leq \beta<t \leq T,\{x, \xi\} \subset \mathbb{R}^{n}, c_{1}<c$ and like as in the estimate (37), $\alpha_{1}^{0}$ is any number from the interval $\left(0, \alpha_{1}\right]$, such that $\alpha_{1}^{0}<3 \alpha_{2}$.

From the representation (33) and the estimates (34)-(36), (40) we get a possibility of differentiation under the signs of integrals in $K_{22}^{\varepsilon}$ and uniformly convergence with respect to $(t, x)$ as $\varepsilon \rightarrow 0$ for any fixed $(\tau, \xi)$. Hence, it is valid the equality

$$
\begin{gathered}
\lim _{\varepsilon \rightarrow 0} K_{2}^{\varepsilon}(t, x ; \tau, \xi)=\int_{\tau}^{t} d \beta \int_{\mathbb{R}^{n}} S Z_{2}(t, x ; \beta, \lambda) \Delta_{\lambda}^{X(t-\beta)} Q_{2}(\beta, \lambda ; \tau, \xi) d \lambda+ \\
\quad+\int_{\tau}^{t}\left(\int_{\mathbb{R}^{n}} S Z_{2}(t, x ; \beta, \lambda) d \lambda\right) Q_{2}(\beta, X(t-\beta) ; \tau, \xi) d \beta .
\end{gathered}
$$

Based on (32) and (41) we have

$$
\begin{gathered}
S_{L} W_{2}=\int_{\tau}^{t} d \beta \int_{\mathbb{R}^{n}} S Z_{2}(t, x ; \beta, \lambda) \Delta_{\lambda}^{X(t-\beta)} Q_{2}(\beta, \lambda ; \tau, \xi) d \lambda+ \\
+\int_{\tau}^{t}\left(\int_{\mathbb{R}^{n}} S Z_{2}(t, x ; \beta, \lambda) d \lambda\right) Q_{2}(\beta, X(t-\beta) ; \tau, \xi) d \beta+Q_{2}(t, x ; \tau, \xi) .
\end{gathered}
$$

Note, that the existence of derivatives $\partial_{x_{1}}^{k_{1}} W_{2},\left|k_{1}\right| \leq 2$, under conditions $\mathbf{1}$ and $\mathbf{2}$ with $\alpha_{1} \in(0,1)$ and $\alpha_{2} \in(0,1 / 3]$ is proved in the paper [32]. Thus, the $L$-FSCP exists under above conditions. So, the following theorem has been proved.

Theorem 2. Let the conditions 1 and 2 with $\alpha_{1} \in(0,1)$ and $\alpha_{2} \in(0,2 / 3)$ be satisfied. Then there exists an L-FSCP $Z$ for the equation (9), and

$$
\begin{gathered}
\left|\partial_{x_{1}}^{k_{1}} Z(t, x ; \tau, \xi)\right| \leq C(t-\tau)^{-M-M_{k 0}} E_{c}(t-\tau, x, \xi), \\
\left|S_{L} Z(t, x ; \tau, \xi)\right| \leq C(t-\tau)^{-M-1} E_{c}(t-\tau, x, \xi),
\end{gathered}
$$

where $0 \leq \tau<t \leq T,\{x, \xi\} \subset \mathbb{R}^{n}, k_{1} \in \mathbb{Z}_{+}^{n_{1}},\left|k_{1}\right| \leq 2$.

7. Conclusions. The review of the papers where the Levi method of parametrix was used for the parabolic equations or systems is done in the article. The features of choice of the parametrix for the parabolic systems with different degenerations and singularities are considered. In particular, for degenerate ultraparabolic equations of the Kolmogorov type a review of results of construction of FSCP under different assumptions on the coefficients is presented and also new authors' results related to the conditions of the existence of a classical FSCP and $L$-FSCP are given. The obtained results could be applied in the research of the correct solvability of the Cauchy problem for linear equations and for local solvability of quasilinear ultraparabolic equations of the Kolmogorov type, and also in the construction of a FSCP for more general degenerate equations of the Kolmogorov type. 


\section{REFERENCES}

1. Levi E.E. Sulle equazioni lineari totalmente ellitiche alle derivate parziali// Rend. Circ. Matem. Palermo. - 1907. - V.24 - P. 275-317.

2. Levi E.E. On linear elliptic partial differential equations// Usp. Mat. Nauk. -1941. - V.8. - P. $249-292$. (in Russian)

3. Shapiro Z.Ya. On elliptic systems of partial differential equations// Soviet Math. Dokl. - 1945. - V.46, №4. - P. 146-149. (in Russian)

4. Lopatynsky Ya.B. Fundamental system of solutions for elliptic system of lineaar differential equations// Ukr. Mat. J. - 1951. - V.3, №1. - P. 3-38. (in Russian)

5. Ivasyshen S.D. On influence of ideas of Ya.B. Lopatynsky on developent of the theory of parabolic systems// Nonlinear Boundary Problems. - 2010. - V.20. - P. 45-53. (in Ukrainian)

6. Dressel F. The fundamental solution of the parabolic equation// Duke Math. J. - 1940. - V.7, №4. P. 186-203.

7. Pogorzelski W. Etude de la solution fondamentale de l'equation parabolique// Ricerche di Mat. - 1956. V.5 - P. 25-57.

8. Aronson D.G. The fundamental solution of a linear parabolic equation containing a small parameter// III. J. Math. - 1959. - V.3. - P. 580-619.

9. Eidelman S.D. Parabolic Systems, North-Holland, Amsterdam, 1969 (Russian edition, Nauka, Moscow, 1964).

10. Eidelman S.D., Ivasyshen S.D., Kochubei A.N. Analytic methods in the theory of differential and pseudodifferential equations of parabolic type. - Operator Theory: Adv. and Appl., 2004, V.152, 390 p.

11. Kolmogorov A.N. Zufällige Bewegungen (Zur Theorie der Brownschen Bewegung)// Ann. Math. - 1934.V.35. - P. 116-117.

12. Weber M. The fundamental solution of a degenerate partial differential equation of parabolic type// Trans. Amer. Math. Soc. - 1951. - V.71. - P. 24-37.

13. Il'in A.M. On a class of ultra-parabolic equations// Soviet Math. Dokl. - 1964. - V.159, №6. - P. 12141217. (in Russian)

14. Sonin I.M. On a class of degenerate diffusion processes// Theor. Probab. Appl. - 1967. - V.12, №3. P. 490-496. (in Russian)

15. Pascucci A. A. Kolmogorov equations in physics and in finance// Progress in Nonlinear Differential Equations and Their Applications. - Basel: Birkhäuser. - 2005. - V.63. - P. 313-324.

16. Ivasyshen S.D., Medynsky I.P. The Fokker-Planck-Kolmogorov equations for some degenerate diffusion processes// Theory of stochastic processes. - 2010. - 16(32), №1. - P. 57-66.

17. Kuptsov L.P. Fundamental solutions of certain degenerate second-order parabolic equations// Math. Notes. - 1982. - V.31, №4. - P. 283-289. (in Russian)

18. Malitskaya A.P. Fundamental solutions for a class of degenerate parabolic equations// Approximate Methods of Integration of Differential and Integral Equations. - Kiev: Kiev. Ped. Inst, - 1973. - P. 109130. (in Russian)

19. Eidelman S.D., Tychinska L.M. Construction of fundamental solutions for some degenerate parabolic equations of an arbitrary order// Dopovidi AN Ukr. SSR, Ser. A. - 1979. - №11. - P. 896-899. (in Ukrainian)

20. Malitskaya A.P. Construction of a fundamental solution for a class of high-order degenerate parabolic equations// Ukr. Math. J. - 1980. - V.32, №6. - P. 508-514. (in Russian)

21. Malitskaya A.P. Structure of the fundamental solutions of some ultraparabolic equations of high order// Ukr. Math. J. - 1985. - V.37, №6. - P. 582-587.

22. Ivasyshen S.D., Tychynskaya L.M., Eidelman S.D. Fundamental solutions of the Cauchi problem for a class of second order ultraparabolic equations// Dopovidi AN Ukr. SSR, Ser. A. - 1990. - №5. - P. 6-8. (in Russian)

23. Ivasyshen S.D., Androsova L.N. Integral representation of solutions of a class of degenerate parabolic Kolmogorov equations// Diff. Equations. - 1991. - V.27, №3. - P. 479-487. (in Russian)

24. Dron' V.S., Ivasyshen S.D. Properties of fundamental solutions of the Cauchi problem for a class of ultraparabolic equations// Ukr. Mat. J. - 1998. - V.50, №11. - P. 1482-1496. (in Ukrainian) 
25. Eidelman S.D., Ivasyshen S.D., Malytska H.P. A modified Levi method: development and application// Dopovidi NAN Ukrainy. - 1998. - №5. - P. 14-19. (in Ukrainian)

26. Dron' V.S. On correct solvability of the Cauchy problem for the ultraparabolic equatin of Kolmogorov type// Mat. Metody Fiz.-Mech. Polya. - 1999. - V.42, №3. - P. 52-55. (in Ukrainian)

27. Ivasyshen S.D., Layuk V.V. Fundamental solutions of the Cauchy problem for some degenerate parabolic equations of Kolmogorov type// Ukr. Mat. J. - 2011. - V.63, №11. - P. 1469-1500. (in Ukrainian)

28. Lanconelli E., Polidoro S. On a class hypoelliptic evolution operators// Rend. Sem. Mat. Univ. Pol. Torino. - 1994. - V.52, №1. - P. 29-63.

29. Polidoro S. On a class of ultraparabolic operators of Kolmogorov-Fokker-Planck type// Le Matematiche. 1994. - V.49, №1. - P. 53-105.

30. Polidoro S. Uniqueness and representation theorems for solution of Komogorov-Fokker-Planck equations// Arch. Rational Mech. Anal. - 1997. - V.137, №4. - P. 321-340.

31. Di Francesco M., Pascucci A. On a class of degenerate parabolic equations of Kolmogorov type// AMRX Appl. Math. Res. Express. - 2005. - №3. - P. 77-116.

32. Ivasyshen S.D., Medynsky I.P. Classical fundamental solutions of the Cauchy problem for ultraparabolic equations of Kolmogorov type with two groops of spartial variables// Proceedings of Institute of Mathematics NAS of Ukraine. - 2016. - V.13, №1. - P. 108-155. (in Ukrainian)

33. Ivasyshen S.D., Medynsky I. P. The classical fundamental solution of a degenerate Kolmogorov's equation with coefficients indepedent on variables of degeneration// Bukov. Mat. J. - 2014. - V.2, №2-3. - P. 94106. (in Ukrainian)

34. Shatyro Ya.I. Inner estimates for solutions of a class of ultra-parabolic equations// Mat. Zapiski Uralsk. Univ. - 1970. - V.7, №4. - P. 131-154. (in Russian)

35. Shatyro Ya.I. On smoothness of solutions of some degenerate second order equations// Mat. Zametki. 1971. - V.10, №1. - P. 101-111. (in Russian)

National Technical University of Ukraine

ivasyshen_sd@mail.ru

National University Lviv Polytechnic

i.p.medynsky@gmail.com 